\title{
Argon excimer emission from high-pressure microdischarges in metal capillaries
}

\author{
R. Mohan Sankaran and Konstantinos P. Giapis \\ Division of Chemistry and Chemical Engineering, California Institute of Technology, \\ Pasadena, California 91125 \\ Mohamed Moselhy and Karl H. Schoenbach \\ Physical Electronics Research Institute, Department of Electrical and Computer Engineering, Old Dominion \\ University, Norfolk, Virginia 23529
}

(Received 25 August 2003; accepted 13 October 2003)

\begin{abstract}
We report on argon excimer emission from high-pressure microdischarges formed inside metal capillaries with or without gas flow. Excimer emission intensity from a single tube increases linearly with gas pressure between 400 and 1000 Torr. Higher discharge current also results in initial intensity gains until gas heating causes saturation or intensity drop. Argon flow through the discharge intensifies emission perhaps by gas cooling. Emission intensity was found to be additive in prealigned dual microdischarges, suggesting that an array of microdischarges could produce a high-intensity excimer source. (C) 2003 American Institute of Physics. [DOI: 10.1063/1.1632034]
\end{abstract}

Microhollow cathode discharges (MHCDs) are highpressure microdischarges formed in holes [inside diameter (i.d.) $\sim 100 \mu \mathrm{m}]$ drilled in thin metal-dielectric-metal films. ${ }^{1}$ These discharges can operate stably at pressures exceeding 1 atm while sustaining a large concentration of high-energy electrons. ${ }^{2}$ These two characteristics make microdischarges attractive as a source of excimer radiation which requires three-body collisions of excited atomic states. Indeed, excimer emission has been observed in microdischarges of $\mathrm{Ne}^{3}$ $\mathrm{Ar}$ and $\mathrm{Xe},{ }^{4} \mathrm{ArF},{ }^{5} \mathrm{XeCl},{ }^{6}$ and XeI. ${ }^{7}$ Limited enhancement in excimer intensity from single MHCDs has been achieved ${ }^{4}$ by increasing gas pressure. Larger gains in emission intensity could be obtained more readily by increasing the total plasma volume, a difficult task with these inherently planar devices. Significant gains in excimer intensity could enable fabrication of cw excimer microlasers. ${ }^{6,8}$

Since high-energy electrons in MHCDs are generated in the cathode fall, a thicker cathode electrode with a deeper hole should automatically provide larger cathode area. Metal capillaries offer an easy solution to fabricating such a device with more internal (cathode) area for plasma expansion. Microdischarges in capillaries can still be operated in the hollow cathode mode and have been used as microreactors for materials processing. ${ }^{9}$ We report here results on their performance as sources of excimer radiation.

The experimental set-up for striking microdischarges in capillary tubes bears similarities with that of MHCDs, as shown in Fig. 1. The electrodes consist of a stainless steel tube $(5 \mathrm{~cm}$ long, i.d. $=178 \mu \mathrm{m})$ and a stainless steel grid, operated as the cathode and anode, respectively. A sapphire washer (i.d. $=203 \mu \mathrm{m}$ ) separates the two electrodes by 381 $\mu \mathrm{m}$. The cathode tube is fitted into a water-cooled copper tube (not shown). Discharges were operated using a positive dc power supply; breakdown voltages were less than $2 \mathrm{kV}$ for all conditions studied. The plasma voltage was monitored using a digital oscilloscope and the current was measured with a resistor in series with the discharge. Spectral measurements in the vacuum-ultraviolet (VUV) were performed us- ing a $0.2 \mathrm{~m}$ McPherson scanning monochromator (Model 302 ), with a grating of 600 grooves $/ \mathrm{mm}$ blazed at $150 \mathrm{~nm}$. The setup was placed in a vacuum chamber and aligned with the inlet of the vacuum monochromator $\left(\mathrm{MgF}_{2}\right.$ window) so that emission spectra could be collected through the grid anode. Before each experiment, the discharge chamber was evacuated using a turbomolecular pump, then back-filled with high-purity argon to the desired pressure. Gas was supplied via a mass flow controller either through a side-port in the vacuum chamber (cross-flow) or through the cathode tube (back-flow).

Emission spectra for argon discharges in a cross-flow of gas are shown in Fig. 2 as a function of ambient pressure. At high pressures ( $>400$ Torr), the spectra are dominated by the argon excimer emission continuum peaking near $128 \mathrm{~nm}$. As the pressure is raised from 400 to 1000 Torr, the intensity of the excimer emission increases. Also evident in the spectra are lines corresponding to atomic oxygen at approximately $130.5 \mathrm{~nm}$ and atomic carbon at 156, 160, and $165 \mathrm{~nm}$. These lines are attributed to impurities in the microdischarge from outgassing of materials used in the device set-up. ${ }^{10}$ Increasing the gas cross-flow did not reduce the contamination. The excimer intensity was also found to increase with discharge current from 2 to $10 \mathrm{~mA}$; beyond this range excessive tube

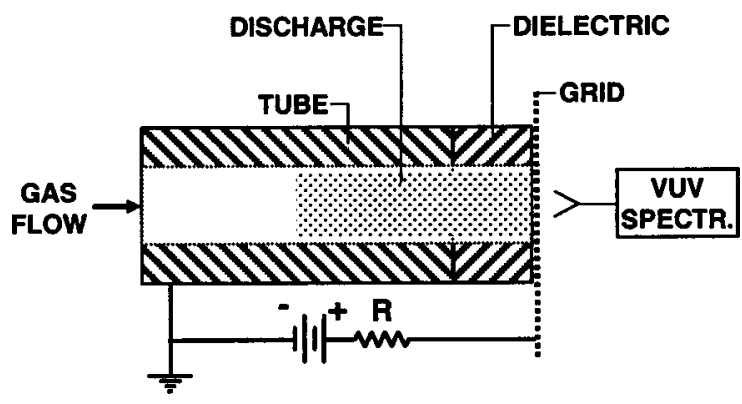

FIG. 1. Schematic of the microdischarge setup (not to scale). The device consists of a stainless steel capillary tube (cathode), a metal grid (anode), and a sapphire spacer washer. VUV spectra were collected from the anode side. Connection to the dc power supply was through a current limiting resistor $(R=100 \mathrm{k} \Omega)$. 


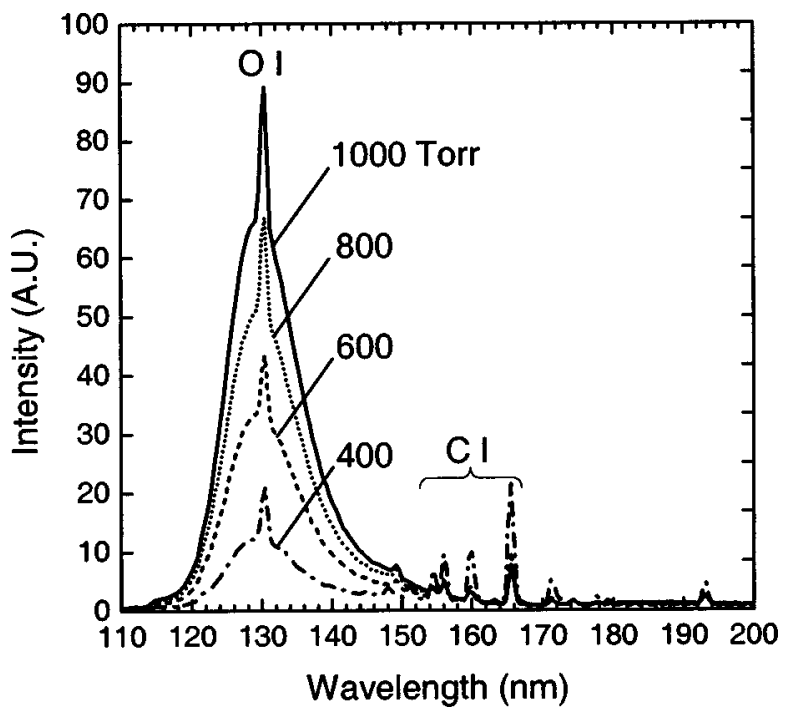

FIG. 2. VUV emission spectra of argon microdischarges in a cathode tube (i.d. $=178 \mu \mathrm{m}$ ) as a function of the ambient gas pressure. The discharge current was held constant at $4 \mathrm{~mA}$. OI and CI refer to lines corresponding to atomic oxygen and carbon states (impurities).

heating ensued (Fig. 3). At 1000 Torr, the optical power which was obtained by integrating the spectra from 115 to $155 \mathrm{~nm}$, increased with current up to $6 \mathrm{~mA}$ and then saturated (Fig. 3). At lower pressures, a maximum was reached at smaller discharge currents followed by a drop in emission, which is attributed to heating ensued.

Cooling of the discharge should be more efficient when gas is flown through the cathode tube (back-flow). Figure 4 shows emission spectra obtained from an argon microdischarge as a function of the flow rate. The discharge was operated at an ambient pressure of 1000 Torr and a current of $4 \mathrm{~mA}$. More intense excimer emission was observed at larger flow rates. Unlike the emission spectra for cross-flow of the gas (Fig. 2), lines from oxygen and carbon contamination were indiscernible even for the lowest flow rate. The absence of emission lines from contaminants is attributed to reduced outgassing due to more efficient cooling. The concomitant increase in the intensity of the excimer emission continuum

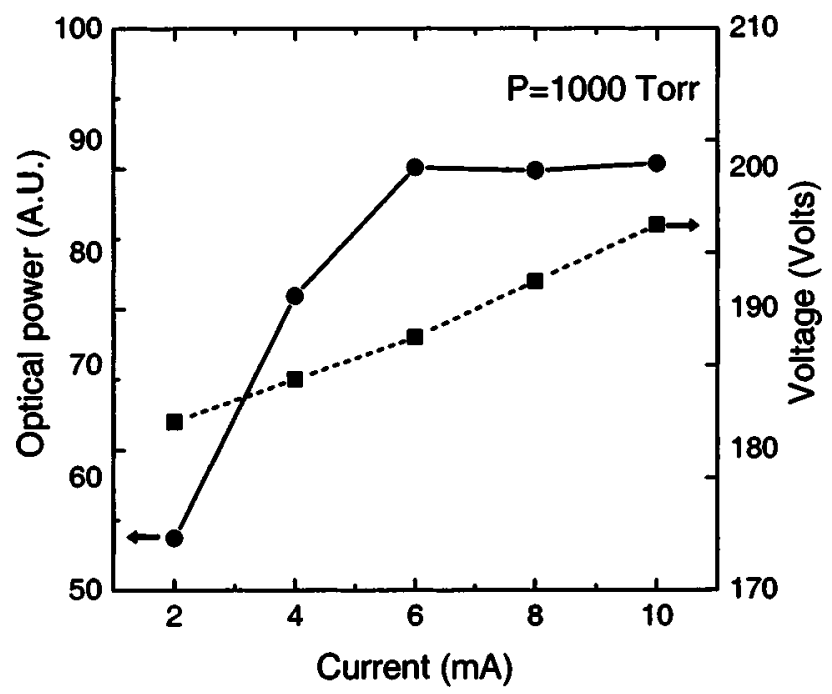

FIG. 3. VUV optical power and discharge voltage as a function of current for a single microdischarge in argon at a pressure of 1000 Torr. Gas was

supplied in a cross-flow configuration.
Downloaded 30 Apr 2006 to 131.215 .240 .9 . Redistribution subject to AIP license or copyright, see http://apl.aip.org/apl/copyright.jsp

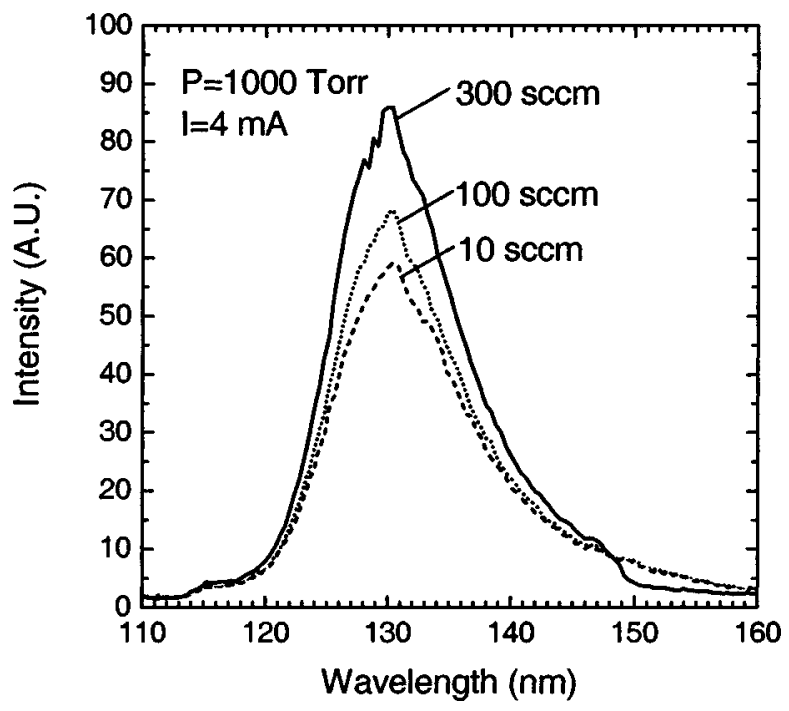

FIG. 4. Argon excimer emission spectra of microdischarges with gas flown through the cathode tube at the indicated flow rates. Ambient gas pressure and discharge current were kept constant at 1000 Torr and $4 \mathrm{~mA}$, respectively.

with flow rate corroborates the role of gas heating and its adverse effect on excimer emission. ${ }^{11}$

Our experiments in single capillary microdischarges suggest that gains in excimer emission intensity are limited despite the dependence on discharge current, gas pressure and flow rate. The positively sloped current-voltage dependence (Fig. 3) indicates operation in the abnormal glow discharge mode. Although the tube geometry provides abundant cathode surface for the plasma to expand, the desired higher discharge current must still pass axially through the tiny tube cross-section. We speculate that the ensuing intense heating (the tube tip can turn red-hot) enhances electron emission at the tube end thus limiting plasma expansion.

An alternative scheme for intensifying the on-axis excimer emission while avoiding heating is to operate aligned arrays of identical microdischarges. The same voltage drop across each tube would sustain a fixed current through the array. The current would be adjusted for maximum excimer emission while minimizing heating problems for a single microdischarge. We tested this idea by introducing a tube segment ( $5 \mathrm{~mm}$ long) between the cathode and the anode of a single microdischarge (see inset, Fig. 5). The supply voltage was increased to permit two discharges to form: two identical plasma potentials must be maintained. Since the current was kept low, tip heating was not excessive and the plasma expansion was not impeded. The total discharge volume was thus doubled. To determine the difference in excimer emission between a single-tube and dual-tube setup, the two discharges were operated sequentially. First, a single discharge was formed in tube 1 (grid is anode); second, a single discharge was formed in tube 2 (tube 1 is anode); finally, both discharges were formed simultaneously (grid is anode, tube 1 is floated). In Fig. 5 we compare spectra for the three cases, collected through a $0.2 \times 10 \mathrm{~mm}$ slit located $6 \mathrm{~mm}$ away; the ambient pressure and discharge current were 1000 Torr and 4 $\mathrm{mA}$, respectively. We find that discharge 1 is about five times more intense than discharge 2. Assuming that these discharges are an assembly of identical point sources extending 


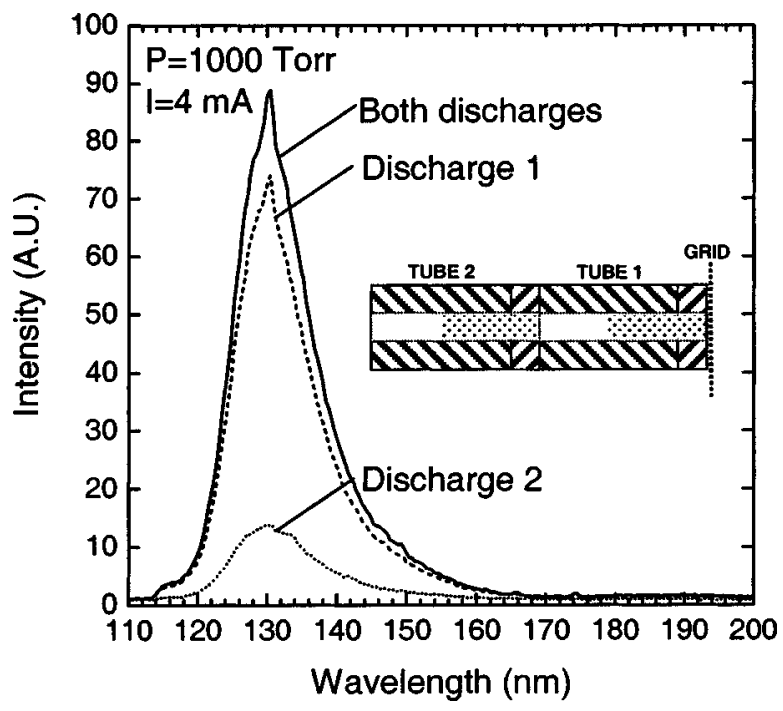

FIG. 5. Argon excimer emission spectra from single-tube and dual-tube microdischarges operated in a sequence described in the text. The inset illustrates the setup schematic. The single-tube discharge was operated at a current of $4 \mathrm{~mA}$ and voltage of $210 \mathrm{~V}$; simultaneous operation of the dualtube was obtained at a current of $4 \mathrm{~mA}$ and voltage of $420 \mathrm{~V}$.

2-3 mm into each tube, geometric considerations suggest that the intensity difference should be a factor of three. Since the front discharge (closer to the monochromator) can be viewed off axis, we expect more light to be collected from discharge 1 through the rectangular slit. The excimer intensity of the dual discharge setup is approximately equal to the sum of the intensities from the individual discharges for pressures between 600 and 1350 Torr (not shown).
Excimer emission has been studied in high-pressure argon microdischarges formed in metal capillary tubes. Emission intensity was found to increase with discharge current, ambient pressure, and argon flow rate through the discharge. However, the intensity gains were limited by gas heating. The additivity of excimer intensity from prealigned dual microdischarges - operated simultaneously at a current such that the intensity of a single discharge is maximumsuggests a scheme for fabricating a source of intense excimer radiation that could lead to a dc excimer microlaser.

We gratefully acknowledge partial support of this work by NSF (CTS-0317397).

${ }^{1}$ K. H. Schoenbach, A. El-Habachi, W. Shi, and M. Ciocca, Plasma Sources Sci. Technol. 6, 468 (1997).

${ }^{2}$ J. W. Frame, D. J. Wheeler, T. A. DeTemple, and J. G. Eden, Appl. Phys. Lett. 71, 1165 (1997).

${ }^{3}$ P. Kurunczi, H. Shah, and K. Becker, J. Phys. B 32, L651 (1999).

${ }^{4}$ A. El-Habachi and K. H. Schoenbach, Appl. Phys. Lett. 72, 22 (1998).

${ }^{5}$ K. H. Schoenbach, A. El-Habachi, M. Moselhy, W. Shi, and R. H. Stark, Phys. Plasmas 7, 2186 (2000).

${ }^{6}$ A. El-Habachi, W. Shi, M. Moselhy, R. H. Stark, and K. H. Schoenbach, J. Appl. Phys. 88, 3220 (2000).

${ }^{7}$ J. W. Frame, P. C. John, T. A. DeTemple, and J. G. Eden, Appl. Phys. Lett. 72, 2634 (1998).

${ }^{8}$ B. A. Vojak, S.-J. Park, C. J. Wagner, and J. G. Eden, Appl. Phys. Lett. 78, 1340 (2001).

${ }^{9}$ R. M. Sankaran and K. P. Giapis, J. Appl. Phys. 92, 2406 (2002).

${ }^{10}$ M. Moselhy, R. H. Stark, K. H. Schoenbach, and U. Kogelschatz, Appl. Phys. Lett. 78, 880 (2001).

${ }^{11}$ J. D. Ametepe, J. Diggs, D. M. Manos, and J. M. Kelley, J. Appl. Phys. 85, 7505 (1999) 